We appreciate the response to this publication feature and welcome all contributions. Contributions may be sent to Jose A. Mascorro, our Technical Editor, at his e-mail address: jmascor@tulane.edu. José may also be reached at the Department of Structural and Cellular Biology, Tulane University Health Sciences Center, 1430 Tulane Ave., New Orleans, LA 70112 and Ph: (504) 584-2747 (504) 584-1687

\section{Technical Note on the Preparation of Un-decalcified Trabecular Bone for Examination by TEM}

\author{
Jeannette Taylor ${ }^{*}$ and Iwona Jasiuk** \\ ${ }^{*}$ Emory University ${ }^{* *}$ Georgia Institute of Technology.
}

Samples of un-decalcified osteoporotic and normal human trabecular bone were prepared for examination by transmission electron microscopy (TEM) to study their ultrastructure. In this note, the samples are referred to as un-decalcified bone rather than simply as bone, because bone is usually decalcified in TEM preparations. Decalcified bone is much easier to cut. Un-decal-
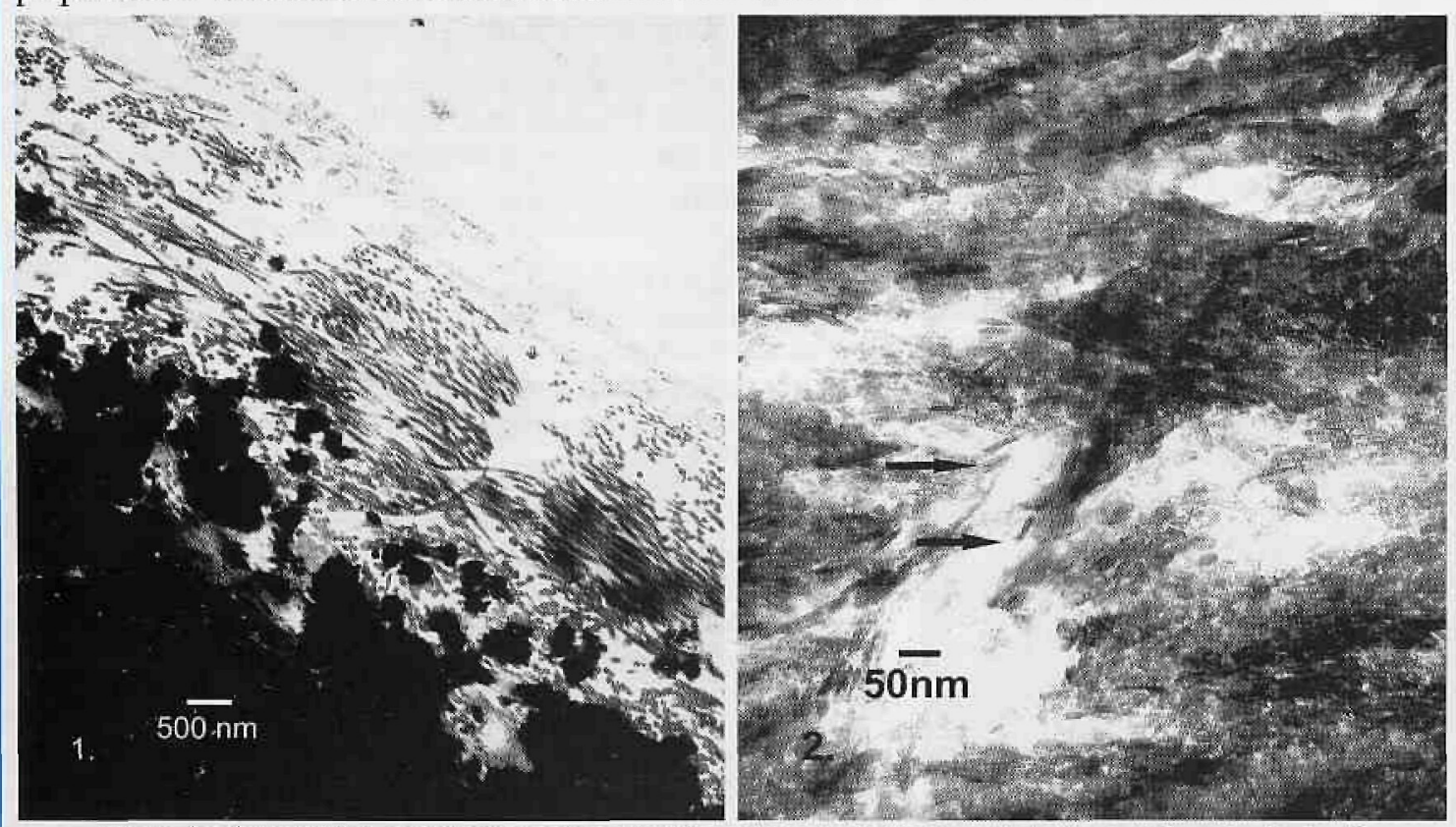

Figure 1 , taken at $10 \mathrm{kX}$, and Figure 2, taken at $80 \mathrm{kX}$, are images of collagen and apatite crystals, respectively. Figure 1 shows areas of collagen only (top left and middle) and of mineralized bone (botrom left). Figure 2 shows the apatite crystals, fragile but wearing and destructive to a glass or dianond knife. The arrows point to a couple of individual crystals. Both micrographs are of osteoporotic trabecular bone in fenoral head. The micrographs were taken with a JEOL JEM-1210 TEM at 90 and $80 \mathrm{kV}$ respectively.

cified bone presents more challenges in sample preparation than decalcified bone because apatite crystals make it harder, stronger and more brittle. The crystals challenge the cutting edge of the knife be it a diamond or glass knife. They will dull a knife and can chip it.

These bone samples were prepared for examination by TEM by cutting them into pieces approximately $1 \mathrm{~mm}^{3}$. They were post fixed in $1 \%$ osmium tetroxide followed by dehydration in an acetone series $(30,50,70,80,90$, and 2 X $100 \%)$. An infiltration of the bone by Spurr's epoxy resin (Spurr, 1969) was accomplished by first applying three changes of acetone and Spurr's resin mixtures under vacuum, followed by three changes of pure Spurr's resin under vacuum, and then followed by embedding and polymerization.
Spurr's epoxy resin was selected because its very low viscosity allowed it to invest the tiny voids in the trabecular bone and because it polymerized well and made a hard block. The bony areas were still harder than the resin and cut at a different rate than the epoxy or any of the softer tissue of the bone. To compensate for this, the sample blocks were cut at the slowest possible speed on the microtome. This reduced the wrinkling that occurs when materials of different densities are cut together in a sample block.

The blocks of tissue were trimmed and faced with glass knives. Selected blocks were ultrathin sectioned ( 90 to $100 \mathrm{~nm}$ ) with a diamond knife on an RMC MT-7000 ultramicrotome.

While cutting the ultrathin sections, the areas of hard bone appeared absent or transparent while the surrounding plastic and soft tissue such as marrow and osteocytes appeared as the usual refractive color on the water of the knife boat indicating section thickness. It appeared as though the hard bone had simply not cut but when sections were placed onto grids with a support film, they were revealed to be present.

Single slot girds with a Formvar ${ }^{m}$ film and three-hundred-mesh grids were selected for this project. Sections supported by Formvar ${ }^{\text {ru }}$ film on slot grids provided views of large areas of the samples without grid bars. This provided a view of the bone ultrastructure, which allowed one to locate areas of interest. Using low magnification images as maps, areas of interest were later located on the 300 mesh grids. Higher magnification images of cell ultrastructure and of the apatite crystals were recorded using the sections on the grids. The mesh grids allowed the best view of the nanostructure of the bone, as there was no film underlying the apatite crystals. A support film would have interfered with the resolution of the apatite crystals.

The goal of this process was to be able to visualize the ultrastructure of osteoporotic and normal human trabecular bone so that they could be compared as to size and arrangement of the apatite crystals and the arrangement of collagen fibrils in lamellar structures. The results of this research are described in Rubin et al. (2003).

\section{References:}

Spurr, A. R. (1969). A low-viscosity epoxy resin embedding medium for electron microscopy, Journal of Ultrastructural Research, 26,31 .

Rubin, M., Jasiuk, I., Taylor, J., Rubin, J., Ganey, T., \& Apkarian, R. (2003). TEM analysis of the nanostructure of normal and osteoporotic human trabecular bone, Bone, 33, 270-282. 